\title{
Investigation on 1-D and 2-D Signal Sparsity Using the Gini Index, L1-Norm and L2-Norm for the Best Sparsity Basis Selection
}

\author{
Y. Parkale and S. Nalbalwar \\ Department of Electronics and Telecommunication Engineering, Dr. BATU., Lonere, Raigad, Maharashtra, India \\ \{yuvrajparkale@gmail.com, slnalbalwar@gmail.com\}
}

\begin{abstract}
Sparsity of signals is a crucial fundamental concept in diverse fields such as compressed sensing, image processing, dictionary learning, blind source separation and sampling theory. The objective of this paper is to present sparsity analysis of 1-D speech signal and 2-D image using Gini index, L1-norm and L2-norm, for the best sparsity basis selection. The DWT families, FFT, DCT, LPC and PCA are used as sparsifying basis. The result shows that the dmey wavelet (1-level decomposition) and bior3.7 wavelet (3-level decomposition) show the greatest value of Gini index for speech. Furthermore, the bior5.5 wavelet shows the lowest value of L1-norm and L2-norm. The DCT exhibits largest Gini index compared to FFT, LPC and PCA for speech. For image signals, the bior3.7 (1-level decomposition) and bior3.1 (3-level decomposition) exhibits highest Gini index. Moreover, the bior3.1 and the bior5.5 wavelet show the lowest value of L1-norm and L2-norm. The PCA exhibits the highest Gini index for image.
\end{abstract}

Keywords: Sparsity, Gini Index, Lorenz Curve, Norms, FFT, DWT, DCT, PCA, Compressed Sensing.

\section{Introduction}

Sparsity is defined as the number of non-zero coefficients in the signal under some transform domain, for a better representation of a signal. The sparsity is a key concept applied in different signal processing areas such as compressed sensing [1, 2, 3], image processing [4, 5], dictionary learning [6], medical imaging [7], sampling theory $[8,9]$, blind source separation $[10,11]$, etc.

There are different methods used for measuring sparsity of a signal. Some of the popular sparsity measures are: $L^{0}, L_{\varepsilon}^{0}, L^{1}, L^{p}, \frac{L^{2}}{L^{1}}, \tanh h_{a, b}, \log$, kurtosis, Hoyer, pq-mean and Gini index $[12,13]$. These methods calculate the values which will represent the sparsity of a signal vector $\vec{u}=\left[u_{1}, u_{2}, \ldots, u_{N}\right]$.

\begin{tabular}{ll}
\hline \multicolumn{2}{c}{ Abbreviations } \\
\hline DFT-Discrete Fourier Transform & PCA-Principal Component Analysis \\
DCT- Discrete Cosine Transform & LPC-Linear Prediction Coding \\
DWT- Discrete Wavelet Transform & CS - Compressed Sensing \\
\hline
\end{tabular}

The $L^{p}$-norm is widely studied and applied sparsity measure. The L0-norm is the traditional sparsity metric which counts the number of non-zero elements in the signal vector given by: $\|\vec{u}\|=u_{j} \neq 0, j=1,2, \ldots, N$. However, it is practically unsuitable because of two disadvantages. The first drawback is the zero derivative of $L^{p}$-norm and thus exhibits zero information. Secondly, it has a very poor performance in the presence of noise. The only way to find the sparsest solution is the exhaustive search method, which is practically NP-hard and thus not used in optimization problems. Hence, L1-norm is most popular sparsity metric. In [12, 13] presented six different attributes of sparsity measures namely: Scaling, Robin Hood, Rising Tide, Bill Gates, Cloning and Babies, and the only measures that are satisfying all of these criteria are pq-means and Gini index.

In many signal processing applications, it is commonly desired to recover the discrete signal with only few numbers of measurements. For example, the success of compressed sensing (CS) $[14,15]$ applications is mainly depends on the sparse or compressible nature of the signal, allowing the efficient acquisition, compression and reconstruction of a signal.

The major contributions of the proposed work are: (1) Presented and investigated different signal sparsity measures such as Gini index (GI), L1-norm and L2-norm. (2) Demonstrate the 1-D speech signal sparsity

B. Iyer, S. Nalbalwar and R.Pawade(Eds.)

ICCASP/ICMMD-2016. Advances in Intelligent Systems Research.

Vol. 137, Pp. 642-651.

(C) 2017- The authors. Published by Atlantis Press

This is an open access article under the CC BY-NC license (http://creativecommons.org/licens)es/by-nc/4). 
analysis with different sparsifying transforms such as Discrete Wavelet Transform (DWT) family, FFT, DCT, LPC and PCA, using sparsity metrics like Gini index (GI), L1-norm and L2-norm. Here, we have investigated for the best sparsity transform for speech signals. (3) Demonstrate the 2-D image signal sparsity analysis with different sparsifying transforms such as Discrete Wavelet Transform (DWT) family, DCT and PCA, with sparsity metrics like Gini index (GI), L1-norm and L2-norm. Here, also we have investigated for the best sparsity transform for 2-D image signals.

The paper is organized as follows: Section 2 introduces the different signal sparsity measurement metrics. Section 3 presents the different sparsifying transform. Section 4 illustrates the experimental results and discussions. Finally, Section 5 presents the conclusions.

\section{Different Signal Sparsity Metrics}

There are different metrics used for the measurement of signal sparsity. The following are some important and commonly used sparsity measures.

\section{A. Gini Index (GI)}

With a vector $\vec{u}=\left[u_{1}, u_{2}, \ldots, u_{N}\right]$, it is ordered from smallest to largest, $u_{(1)} \leq u_{(2)} \leq \cdots \leq u_{(N)}$. Where (1), (2)... (N) are the newest indices after the sorting operation. The Gini index is given by equation (1) as follows:

$$
S(\vec{u})=1-2 \sum_{j=1}^{N} \frac{u_{(j)}}{\|\vec{u}\|_{1}}\left(\frac{N-j+\frac{1}{2}}{N}\right)
$$

The Gini index $[16,17,18,19,20,21]$ is defined as double the area between the Lorenz curve and the equality diagonal. The Fig. 1 shows the graphical interpretation of the Gini index (Double the yellow area). The Gini index can have a value anywhere from 0 to 1 . The perfect equity is indicated by zero Gini index and 1 shows perfect inequity. The larger ratio indicates the more inequitable distribution of the signal sparsity. Hence, a large value of Gini index indicates more signal sparsity. The Gini index shows the best property, where the 45 degrees line (in red) indicates the minimum sparse distribution, i.e. almost all the entries being equal.

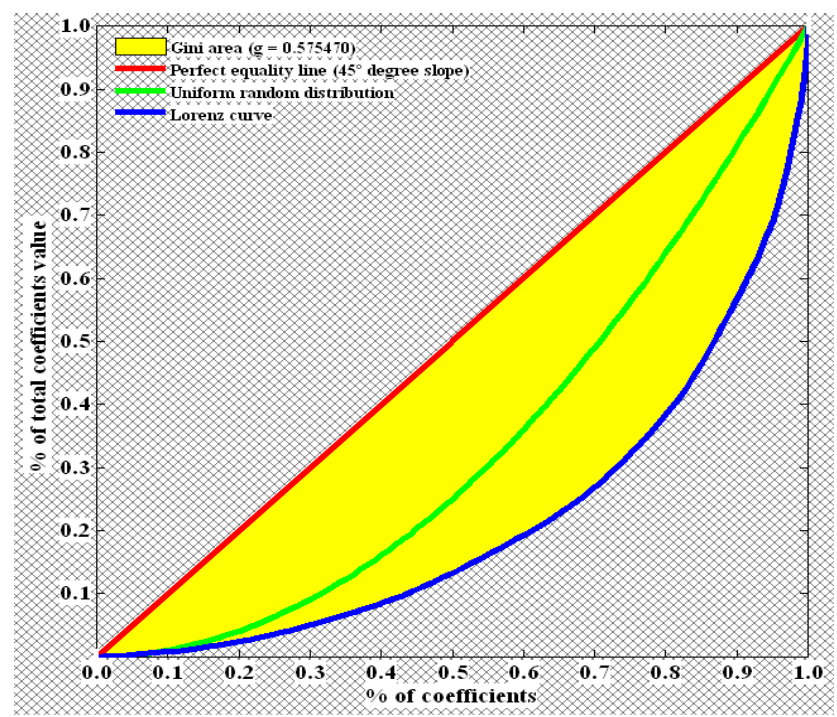

Fig. 1. Graphical interpretation of the Gini index (Double the yellow area)

\section{B. L1-norm}

The L1-norm is calculated by summing the absolute values of all the coefficients of a signal and is given by: 


$$
\|u\|_{1}=\sum_{j} u_{j}
$$

The smallest value of addition indicates the maximum signal sparsity. The L1-norm is a more convex function compared to other norms, and hence commonly used in solving optimization problems. The Fig. 2 shows the diamond shaped geometrical interpretation of L1-norm. The point at which the red line (solution plane) touches to the diamond is called as exact sparse solution. Hence, it is widely used in the optimization to find out the exact sparse solution.

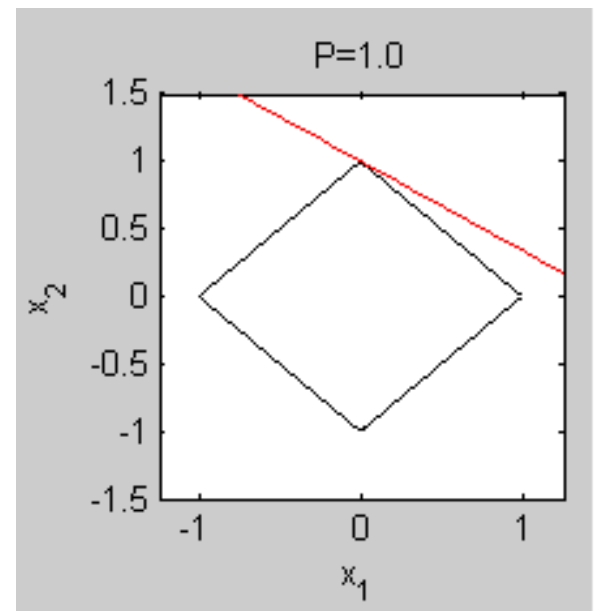

Fig. 2. Geometrical interpretation of L1norm

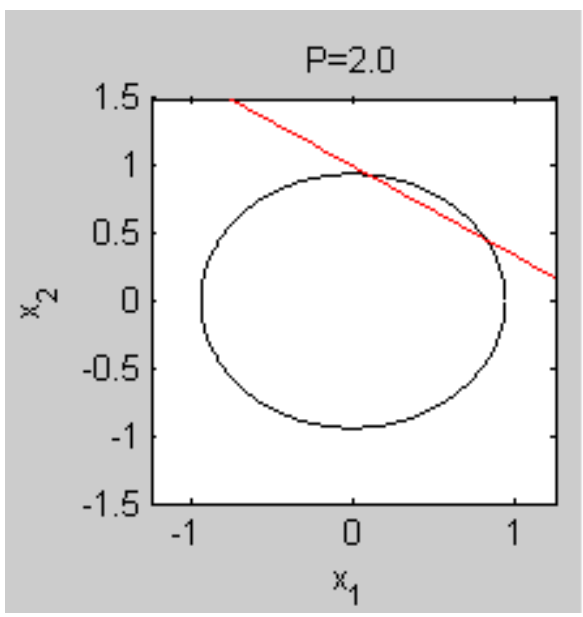

Fig. 3. Geometrical interpretation of L2norm

\section{L2-norm}

The L2-norm will not provide the exact sparse solution as shown by the geometrical interpretation of L2-norm in Fig. 3 and is calculated as follow:

$$
\|u\|_{2}=\left(\sum_{j} u_{j}^{2}\right)^{\frac{1}{2}}
$$

\section{Scarifying Transform}

There are different scarifying transform used for the representation of signal. Some important transforms are discussed as below.

\section{A. Discrete Fourier Transform (DFT)}

The DFT is the basic sparsity transform for a signal of length $n$. But, DFT suffers from a drawback of huge number of additions and multiplication operations, resulting in increased computational cost. The Fast Fourier Transform (FFT) algorithm is used instead of DFT because of the low computational complexity of FFT i.e. $O(n$ $\log n$ ) instead of $O\left(n^{2}\right)$. However, the FFT have drawbacks like applying CS to a complex valued signal is a complex and weariness process. Also, FFT calculates both positive as well as negative side frequencies [22]. For input signal $x$ the DFT is given by: 


$$
\operatorname{DFT} Y(k)=\sum_{n=0}^{N-1} x(n) e^{-j 2 \pi n k / N}
$$
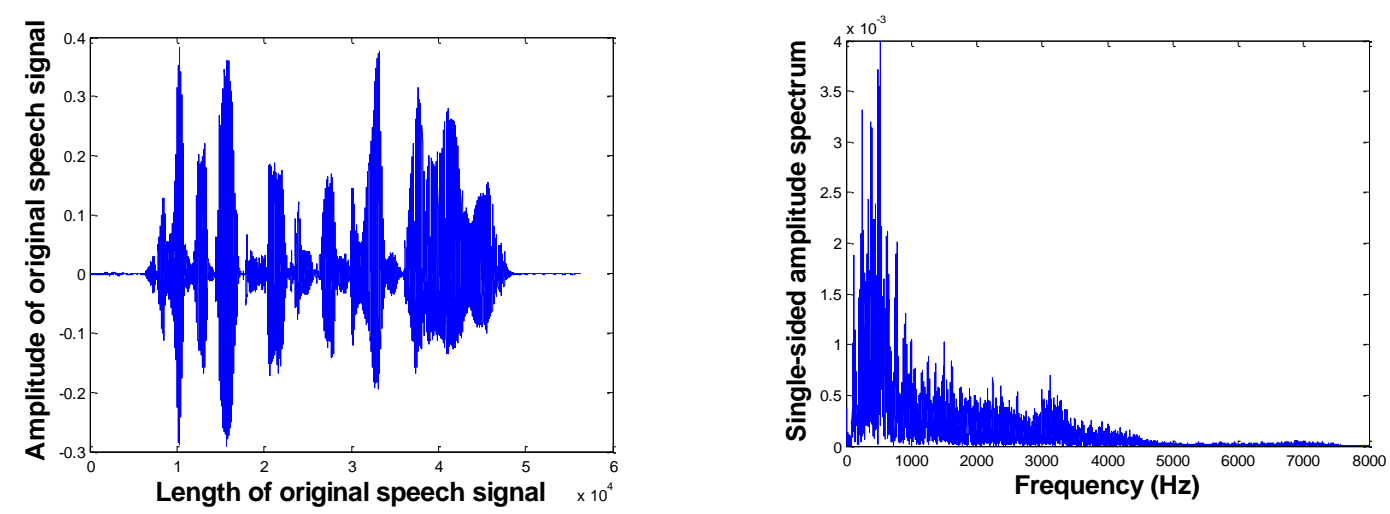

The Fig. 4 shows the original speech signal of length $(\mathrm{N}=56273)$ and Fig. 5 shows the DFT of speech signal.

\section{B. Discrete Cosine Transform (DCT)}

The drawbacks of the FFT can be overcome using the DCT which has excellent energy compaction for highly correlated data. The DCT eliminate the redundancy among the neighboring values, and thus generate transform coefficients which are uncorrelated and encoded independently. Unlike the FFT, the DCT packs most of the signal energy into a few numbers of coefficients. Also, the DCT is a member of a family of real-valued discrete sinusoidal unitary transformation [23]. One of the measures of sparsity is Gini index and DCT shows high Gini index (GI=0.7387). The Fig. 6 shows the DCT of speech signal of length $(\mathrm{N}=56273)$. It has very few numbers of non-zero coefficients. The speech signal sparsity can be increased by thresholding the non-significant coefficients. The DCT of signal $x$ is given as:

$$
\operatorname{DCT}(k)=\alpha(k) \sum_{n=0}^{N-1} x(n) \cos (\pi(2 n-1)(k-1) / 2 N)
$$

Where,

$$
\alpha(k)=\left\{\begin{array}{cc}
1 / \sqrt{N} & \text { for } k=1 \\
\sqrt{2 / N} & \text { for } 2 \leq k \leq N
\end{array}\right.
$$

\section{Wavelet Transform}

The wavelet transform (WT) $[24,25,26]$ gives both the time resolution and frequency resolution of the signal. The wavelet transform is used to overcome the drawbacks of the short-time Fourier transform (STFT), i.e. for the analysis of the non-stationary signals. The STFT shows a constant resolution, while the wavelet transform shows a multi-resolution with respect to frequencies. The wavelet transform gives the approximations of the signal with big wavelets, while small details are given by small scales. The basic idea of the wavelet transform is to represent the signal to be analyzed as a superposition of wavelets. The wavelet transform is a most popular signal analysis tool used in different application areas such as speech or audio and image compression. There are different discrete wavelet transform (DWT) families available for representation of the signal like: Haar, Daubechies, Biorthogonal, Reverse Biorthogonal, Coiflets, Symlets, etc. the Fig. 7 shows the DWT of speech signal of length $(\mathrm{N}=56273)$ with approximation and details. 


\section{Linear Prediction Coding (LPC)}

The Linear predictive coding (LPC) [27] is a fundamental and most powerful speech analysis tool used for representation of the speech spectral envelope in the compressed form. The LPC not only provides the sparse coefficients, but also it gives formants, pitch, an estimate of the intensity and the frequency of the buzz, and the residual signal, which contains most of the speech information. The LPC has applications in speech compression, modeling, representation, etc [28, 29]. The Fig. 8 shows the LPC of speech signal of length $(\mathrm{N}=56273)$.

\section{E. Principal Component Analysis (PCA)}

The Principal component analysis $[30,31]$ is a widely used dimensionality reduction technique having large sets of data e.g. microarray analysis. The main objective of PCA is to reduce the feature space dimensionality while keeping data variance. In PCA, the first covariance matrix of the signal is computed, and then Eigen values and the Eigen vectors of the covariance matrix are calculated. The Eigen vectors corresponding to the largest Eigen values are selected and used for projecting feature space to low dimension space. Thus, the large amount of signal energy is packed in few principal components of PCA which are orthogonal to each other's and thus provides the required signal sparsity [32]. The Fig. 9 shows the PCA of speech signal of length (N=56273).

\section{Experimental Results and Discussions}

\section{- Research Methodology}

The experimentation is performed on the two test signals namely: 1-D speech signal and 2-D image. The test speech signal is available on the CMU/CSTR KDT US English TIMIT database for speech synthesis by Carnegie Mellon University and Edinburgh University [33]. The details of speech file are as follows: File name: Kdt_001.wav, Bit rate: $256 \mathrm{kbps}$, Audio sample rate: $16 \mathrm{kHz}$, Total Duration: 3 seconds. The second test signal is $210 \times 210$ Brain MRI image. The simulation results are generated on MATLAB 7.8.0 (R2009A) with INTEL (R) CORE 2 DUO CPU, 3 GB RAM.

\subsection{1-D Speech Signal Sparsity Analysis using Gini Index, L1-norm and L2-norm}

In this section we have presented the analysis of speech signal sparsity with different sparsity transforms such as Discrete cosine transform(DCT), Fast Fourier transform (FFT), Linear Prediction coding (LPC), Principal component analysis (PCA) and discrete wavelet transform(DWT) families like: Haar, Daubechies, Biorthogonal, Reverse Biorthogonal, Coiflets, Symlets, etc. The sparsity analysis is performed using different sparsity measures such as Gini Index, L1-norm and L2-norm. Furthermore, the greater value of Gini index indicates the greater sparsity of the speech signal, whereas for L1-norm lowest the value, greater is the sparsity of a signal. Also, for L2 -norm smaller the value, sparser is the signal.

The Table 1 shows, the speech signal sparsity analysis of the Discrete Wavelet Transform (DWT) family using Gini Index, L1-norm and L2-norm. The result shows that, the bior3.7 wavelet (3-level decomposition) exhibits the greatest value of the Gini index (GI=0.8737). The bior 3.5 and the bior 3.9 wavelet with GI $=0.8732$ could be the second best choice. The bior5.5 wavelet shows the lowest value of the L1-norm (592.6113) and L2-norm (7.7235). The rbio2.8 wavelet with L1-norm $=656.0824$ and the Rbio3.9 with L2-norm=8.1784 will be the second alternatives.

For the DWT with 1-level decomposition, the Table 1 shows that the dmey wavelet exhibits the greatest value of Gini index (i.e. GI $=0.8208$ ). The bior3.9 wavelet with $\mathrm{GI}=0.8201$ and the bior 3.7 with $\mathrm{GI}=0.8200$ could be the second best choices as there is a very small difference in their values. From the Table $\mathbf{1}$ it is also seen that the bior5.5 wavelet shows the lowest value of the L1-norm (873.4284) and L2-norm (9.3710). The dmey wavelet with L1-norm=882.3817 and the Rbio3.9 with L2-norm=9.4742 could be the second alternatives for speech signal sparsity.

The result shows that the sparsity level of signal increases as there is an increase in the decomposition level of wavelet family. Hence, the DWT family with 3-level decomposition results in increase in the sparsity level of signal compared to the DWT family with 1-level decomposition.

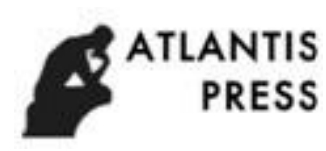


Table 1. A comparative analysis of speech signal sparsity for Discrete Wavelet Transform (DWT) family using the Gini index, L1-norm and L2-norm

\begin{tabular}{|c|c|c|c|c|c|c|c|}
\hline \multirow[t]{2}{*}{$\begin{array}{l}\text { Sr. } \\
\text { no. }\end{array}$} & \multirow[t]{2}{*}{$\begin{array}{l}\text { Sparsifying } \\
\text { Transform }\end{array}$} & \multicolumn{3}{|c|}{$\begin{array}{l}\text { Sparsity measures with 3-level } \\
\text { decomposition }\end{array}$} & \multicolumn{3}{|c|}{$\begin{array}{l}\text { Sparsity measures with 1-level } \\
\text { decomposition }\end{array}$} \\
\hline & & $\begin{array}{l}\text { Gini } \\
\text { index } \\
\text { (GI) }\end{array}$ & $\begin{array}{l}\text { L1- } \\
\text { norm }\end{array}$ & $\begin{array}{l}\text { L2- } \\
\text { norm }\end{array}$ & $\begin{array}{l}\text { Gini } \\
\text { index } \\
\text { (GI) }\end{array}$ & $\begin{array}{l}\text { L1- } \\
\text { norm }\end{array}$ & $\begin{array}{l}\text { L2- } \\
\text { norm }\end{array}$ \\
\hline 1. & $\mathrm{db} 1$ & 0.8008 & 809.6920 & 9.7816 & 0.7713 & 972.8646 & 9.7816 \\
\hline 2. & $\mathrm{db} 2$ & 0.8273 & 737.3517 & 9.7816 & 0.7907 & 932.2936 & 9.7816 \\
\hline 3. & $\mathrm{db} 3$ & 0.8362 & 710.4047 & 9.7816 & 0.7983 & 917.5741 & 9.7816 \\
\hline 4. & $\mathrm{db} 4$ & 0.8410 & 705.2055 & 9.7816 & 0.8024 & 909.8447 & 9.7816 \\
\hline 5. & $\mathrm{db} 5$ & 0.8437 & 696.4810 & 9.7816 & 0.8050 & 905.1808 & 9.7816 \\
\hline 6. & db6 & 0.8461 & 694.9384 & 9.7816 & 0.8072 & 901.8289 & 9.7816 \\
\hline 7. & $\mathrm{db} 7$ & 0.8479 & 689.9088 & 9.7816 & 0.8089 & 899.5069 & 9.7816 \\
\hline 8. & $\mathrm{db} 8$ & 0.8492 & 688.8183 & 9.7816 & 0.8101 & 897.7361 & 9.7816 \\
\hline 9. & db9 & 0.8508 & 685.0001 & 9.7816 & 0.8113 & 895.6545 & 9.7816 \\
\hline 10. & $\mathrm{db} 10$ & 0.8521 & 683.6133 & 9.7816 & 0.8127 & 893.4349 & 9.7816 \\
\hline 11. & sym2 & 0.8273 & 737.3517 & 9.7816 & 0.7907 & 932.2936 & 9.7816 \\
\hline 12. & sym3 & 0.8362 & 710.4047 & 9.7816 & 0.7983 & 917.5741 & 9.7816 \\
\hline 13. & sym4 & 0.8397 & 703.3294 & 9.7816 & 0.8018 & 912.0829 & 9.7816 \\
\hline 14. & sym5 & 0.8431 & 703.1120 & 9.7816 & 0.8043 & 907.2436 & 9.7816 \\
\hline 15. & sym6 & 0.8453 & 695.4730 & 9.7816 & 0.8067 & 903.6103 & 9.7816 \\
\hline 16. & sym7 & 0.8481 & 695.9099 & 9.7816 & 0.8088 & 899.0634 & 9.7816 \\
\hline 17. & sym8 & 0.8495 & 689.1862 & 9.7816 & 0.8099 & 898.2663 & 9.7816 \\
\hline 18. & bior1.1 & 0.8008 & 809.6920 & 9.7816 & 0.7713 & 972.8646 & 9.7816 \\
\hline 19. & bior1.3 & 0.8077 & 868.0260 & 10.7937 & 0.7723 & 986.2193 & 9.9701 \\
\hline 20. & bior1.5 & 0.8093 & 883.0841 & 11.1084 & 0.7725 & 989.9144 & 10.0183 \\
\hline 21. & bior 2.2 & 0.8514 & 761.7358 & 11.3412 & 0.8052 & 925.4935 & 10.0261 \\
\hline 22. & bior2.4 & 0.8527 & 780.7527 & 11.6811 & 0.8054 & 929.6822 & 10.0861 \\
\hline 23. & bior2.6 & 0.8528 & 792.3741 & 11.8326 & 0.8055 & 932.0107 & 10.1184 \\
\hline 24. & bior2.8 & 0.8532 & 801.8361 & 11.9295 & 0.8056 & 933.5522 & 10.1396 \\
\hline 25. & bior3.1 & 0.8712 & 858.0835 & 14.1585 & 0.8192 & 921.7847 & 10.2518 \\
\hline 26. & bior 3.3 & 0.8731 & 847.3781 & 13.7606 & 0.8196 & 926.0613 & 10.3253 \\
\hline 27. & bior3.5 & 0.8732 & 849.4339 & 13.6860 & 0.8198 & 928.6592 & 10.3676 \\
\hline 28. & bior3.7 & 0.8737 & 851.2708 & 13.7478 & 0.8200 & 930.4188 & 10.3963 \\
\hline 29. & bior3.9 & 0.8732 & 858.0313 & 13.8912 & 0.8201 & 931.7157 & 10.4175 \\
\hline 30. & bior4.4 & 0.8440 & 661.8385 & 9.2328 & 0.8070 & 894.2663 & 9.6635 \\
\hline 31. & bior5.5 & 0.8334 & 592.6113 & 7.7235 & 0.8056 & 873.4284 & 9.3710 \\
\hline 32. & bior6. 8 & 0.8530 & 692.7291 & 9.9413 & 0.8120 & 898.5766 & 9.8258 \\
\hline 33. & rbio 1.1 & 0.8008 & 809.6920 & 9.7816 & 0.7713 & 972.8646 & 9.7816 \\
\hline 34. & rbio1.3 & 0.8321 & 682.6080 & 9.1578 & 0.7975 & 910.6444 & 9.6648 \\
\hline 35. & rbio1.5 & 0.8402 & 662.1787 & 9.0993 & 0.8048 & 896.7190 & 9.6476 \\
\hline 36. & rbio 2.2 & 0.8017 & 785.1192 & 9.2516 & 0.7731 & 962.5368 & 9.6595 \\
\hline 37. & rbio2.4 & 0.8168 & 695.3644 & 8.6318 & 0.7878 & 923.2466 & 9.5727 \\
\hline 38. & rbio2.6 & 0.8245 & 668.6529 & 8.4854 & 0.7948 & 907.3536 & 9.5451 \\
\hline 39. & rbio2.8 & 0.8293 & 656.0824 & 8.4273 & 0.7994 & 897.9255 & 9.5311 \\
\hline 40. & rbio3.1 & 0.7618 & 1325.5 & 13.3158 & 0.7312 & 1171 & 10.5086 \\
\hline 41. & rbio3.3 & 0.7905 & 845.4423 & 9.3851 & 0.7638 & 990.4825 & 9.6671 \\
\hline 42. & rbio3.5 & 0.8006 & 748.0424 & 8.6295 & 0.7753 & 951.5696 & 9.5507 \\
\hline 43. & bio3.7 & 0.8076 & 710.6927 & 8.3486 & 0.7823 & 931.5568 & 9.5015 \\
\hline 44. & bio3.9 & 0.8125 & 688.2152 & 8.1784 & 0.7875 & 918.4846 & 9.4742 \\
\hline 45. & rbio4.4 & 0.8352 & 768.2654 & 10.4786 & 0.7954 & 934.6663 & 9.9147 \\
\hline 46. & rbio5.5 & 0.8519 & 855.4873 & 12.7699 & 0.8028 & 948.2945 & 10.2891 \\
\hline 47. & rbio6.8 & 0.8419 & 704.3037 & 9.6705 & 0.8044 & 904.8827 & 9.7429 \\
\hline 48. & coif1 & 0.8279 & 732.9013 & 9.7816 & 0.7913 & 931.3377 & 9.7816 \\
\hline 49. & coif2 & 0.8410 & 705.4458 & 9.7816 & 0.8025 & 910.9446 & 9.7816 \\
\hline 50. & coif3 & 0.8470 & 690.7668 & 9.7816 & 0.8075 & 902.4424 & 9.7816 \\
\hline 51. & coif4 & 0.8506 & 690.4547 & 9.7816 & 0.8108 & 897.2527 & 9.7816 \\
\hline 52. & coif5 & 0.8532 & 680.7731 & 9.7816 & 0.8131 & 893.6535 & 9.7816 \\
\hline 53. & dmey & 0.8621 & 669.1432 & 9.7816 & 0.8208 & 882.3817 & 9.7816 \\
\hline
\end{tabular}


Table 2. A comparative analysis of speech signal sparsity for DCT, FFT, LPC and PCA using the Gini Index, L1norm and L2-norm

\begin{tabular}{cllll}
\hline $\begin{array}{l}\text { Sr. } \\
\text { no. }\end{array}$ & $\begin{array}{l}\text { Sparsifying } \\
\text { Transform }\end{array}$ & Gini index (GI) & L1-norm & L2-norm \\
\hline 1. & DCT & $\mathbf{0 . 7 3 8 7}$ & $\mathbf{1 0 0 1 . 7}$ & $\mathbf{9 . 7 8 1 6}$ \\
2. & FFT & 0.7026 & 263560 & 2320.4 \\
3. & LPC & 0.5002 & 13390 & 110.5893 \\
4. & PCA & 0.6954 & 1200 & $\mathbf{9 . 7 8 1 6}$ \\
\hline
\end{tabular}

The speech signal sparsity analysis for the DCT, FFT, LPC and PCA using Gini Index, L1-norm and L2-norm is shown by the Table 2 . From the Table $\mathbf{2}$ it is observed that the DCT exhibits the largest Gini index (GI=0.7387). The FFT with GI=0.7026 will be the second choice. It is also seen from Table 2 that the lowest value of the L1-norm (1001.7) and L2-norm (9.7816) are shown by the DCT. The PCA could be the second choice with the lowest value of L1-norm (1200) and the L2-norm (9.7816).

\subsection{2-D Image Signal Sparsity Analysis using Gini Index, L1-norm and L2-norm}

This section presents the 2-D image (as shown in Fig. 10) sparsity analysis for the different sparsity transforms such as Discrete cosine transform (DCT), Fast Fourier transform(FFT), Linear Prediction coding (LPC), Principal component analysis (PCA) and discrete wavelet transform(DWT) families like: Haar, Daubechies, Biorthogonal, Reverse Biorthogonal, Coiflets, Symlets, etc.

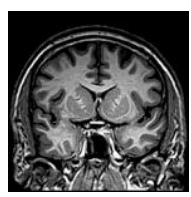

Fig. 10. 2-D test image signal (Example: MRI Coronal Brain.JPG)

Table 3. A comparative analysis of 2-D image sparsity for Discrete Wavelet Transform (DWT) family using the Gini Index, L1-norm and L2-norm (Example Brain MRI)

\begin{tabular}{|c|c|c|c|c|c|c|c|}
\hline \multirow[t]{2}{*}{$\begin{array}{l}\text { Sr. } \\
\text { no. }\end{array}$} & \multirow[t]{2}{*}{$\begin{array}{l}\text { Sparsifying } \\
\text { Transform }\end{array}$} & \multicolumn{3}{|c|}{$\begin{array}{l}\text { Sparsity measures with 3-level } \\
\text { decomposition }\end{array}$} & \multicolumn{3}{|c|}{$\begin{array}{l}\text { Sparsity measures with } 1 \text {-level } \\
\text { decomposition }\end{array}$} \\
\hline & & $\begin{array}{l}\text { Gini } \\
\text { index } \\
\text { (GI) }\end{array}$ & $\begin{array}{l}\text { L1- } \\
\text { Norm }\end{array}$ & $\begin{array}{l}\text { L2- } \\
\text { norm }\end{array}$ & $\begin{array}{l}\text { Gini } \\
\text { index } \\
\text { (GI) }\end{array}$ & $\begin{array}{l}\text { L1- } \\
\text { norm }\end{array}$ & $\begin{array}{l}\text { L2- } \\
\text { norm }\end{array}$ \\
\hline 1. & $\mathrm{db} 1$ & 0.9186 & 1848500 & 19945 & 0.8165 & 2398000 & 19945 \\
\hline 2. & $\mathrm{db} 2$ & 0.9211 & 1824100 & 19945 & 0.8211 & 2359200 & 19945 \\
\hline 3. & $\mathrm{db} 3$ & 0.9231 & 1801100 & 19945 & 0.8218 & 2346100 & 19945 \\
\hline 4. & $\mathrm{db} 4$ & 0.9227 & 1805100 & 19945 & 0.8219 & 2346300 & 19945 \\
\hline 5. & $\mathrm{db} 5$ & 0.9230 & 1806100 & 19945 & 0.8222 & 2346700 & 19945 \\
\hline 6. & $\mathrm{db} 6$ & 0.9228 & 1807600 & 19945 & 0.8229 & 2343100 & 19945 \\
\hline 7. & $\mathrm{db} 7$ & 0.9227 & 1809100 & 19945 & 0.8236 & 2337600 & 19945 \\
\hline 8. & $\mathrm{db} 8$ & 0.9225 & 1813700 & 19945 & 0.8238 & 2338100 & 19945 \\
\hline 9. & db9 & 0.9221 & 1820100 & 19945 & 0.8236 & 2341800 & 19945 \\
\hline 10. & $\mathrm{db} 10$ & 0.9218 & 1828200 & 19945 & 0.8232 & 2345900 & 19945 \\
\hline 11. & sym2 & 0.9211 & 1824100 & 19945 & 0.8211 & 2359200 & 19945 \\
\hline 12. & sym3 & 0.9231 & 1801100 & 19945 & 0.8218 & 2346100 & 19945 \\
\hline 13. & sym4 & 0.9240 & 1787800 & 19945 & 0.8233 & 2334200 & 19945 \\
\hline 14. & sym5 & 0.9224 & 1802500 & 19945 & 0.8230 & 2338500 & 19945 \\
\hline 15. & sym6 & 0.9244 & 1783600 & 19945 & 0.8240 & 2329000 & 19945 \\
\hline 16. & sym7 & 0.9225 & 1802500 & 19945 & 0.8222 & 2342500 & 19945 \\
\hline 17. & sym8 & 0.9241 & 1785200 & 19945 & 0.8242 & 2327500 & 19945 \\
\hline 18. & bior1.1 & 0.9186 & 1848500 & 19945 & 0.8165 & 2398000 & 19945 \\
\hline 19. & bior1.3 & 0.9168 & 1912200 & 20425 & 0.8190 & 2407300 & 20119 \\
\hline 20. & bior1.5 & 0.9152 & 1946100 & 20613 & 0.8198 & 2413100 & 20178 \\
\hline 21. & bior2.2 & 0.9299 & 1797500 & 20904 & 0.8293 & 2316300 & 20203 \\
\hline 22. & bior2.4 & 0.9288 & 1823900 & 21030 & 0.8299 & 2327100 & 20277 \\
\hline 23. & bior2.6 & 0.9272 & 1845700 & 21094 & 0.8301 & 2333400 & 20315 \\
\hline
\end{tabular}




\begin{tabular}{llllllll}
\hline \hline 24. & bior2.8 & 0.9263 & 1859400 & 21138 & 0.8303 & 2337600 & 20339 \\
25. & bior3.1 & $\mathbf{0 . 9 3 5 5}$ & 1969800 & 24035 & 0.8340 & $\mathbf{2 2 9 2 6 0 0}$ & 20440 \\
26. & bior3.3 & 0.9319 & 1923900 & 22904 & 0.8344 & 2310300 & 20549 \\
27. & bior3.5 & 0.9301 & 1934500 & 22771 & 0.8346 & 2319500 & 20601 \\
28. & bior3.7 & 0.9302 & 1941600 & 22813 & $\mathbf{0 . 8 3 4 7}$ & 2325700 & 20633 \\
29. & bior3.9 & 0.9295 & 1955000 & 22859 & 0.8347 & 2330300 & 20656 \\
30. & bior4.4 & 0.9256 & 1745100 & 19746 & 0.8232 & 2312400 & 19841 \\
31. & bior5.5 & 0.9221 & $\mathbf{1 7 3 3 9 0 0}$ & $\mathbf{1 9 1 0 2}$ & 0.8160 & 2338600 & $\mathbf{1 9 5 7 0}$ \\
32. & bior6.8 & 0.9255 & 1772000 & 20039 & 0.8253 & 2315700 & 19973 \\
33. & rbio 1.1 & 0.9186 & 1848500 & 19945 & 0.8165 & 2398000 & 19945 \\
34. & rbio1.3 & 0.9246 & 1754400 & 19688 & 0.8217 & 2330900 & 19841 \\
35. & rbio1.5 & 0.9245 & 1750600 & 19662 & 0.8224 & 2320700 & 19828 \\
36. & rbio2.2 & 0.9062 & 1979500 & 19869 & 0.8073 & 2464800 & 19867 \\
37. & rbio2.4 & 0.9145 & 1864700 & 19565 & 0.8128 & 2401000 & 19756 \\
38. & rbio2.6 & 0.9163 & 1837500 & 19491 & 0.8142 & 2384000 & 19727 \\
39. & rbio2.8 & 0.9169 & 1829600 & 19466 & 0.8149 & 2376600 & 19715 \\
40. & rbio3.1 & 0.8529 & 2674500 & 22580 & 0.7712 & 2805000 & 20884 \\
41. & rbio3.3 & 0.8903 & 2168000 & 20415 & 0.7959 & 2548300 & 19973 \\
42. & rbio3.5 & 0.8992 & 2.050300 & 19964 & 0.8016 & 2492400 & 19816 \\
43. & bio3.7 & 0.9027 & 2003700 & 19772 & 0.8039 & 2470300 & 19760 \\
44. & bio3.9 & 0.9050 & 1975400 & 19667 & 0.8050 & 2459000 & 19731 \\
45. & rbio4.4 & 0.9253 & 1905900 & 21368 & 0.8220 & 2374800 & 20071 \\
46. & rbio5.5 & 0.9208 & 1823200 & 19912 & 0.8295 & 2370300 & 20480 \\
47. & rbio6.8 & 0.9216 & 1821400 & 19945 & 0.8219 & 2348100 & 19925 \\
48. & coif1 & 0.9234 & 1793600 & 19945 & 0.8206 & 2363500 & 19945 \\
49. & coif2 & 0.9238 & 1789400 & 19945 & 0.8231 & 2336200 & 19945 \\
50. & coif3 & 0.9238 & 1789600 & 19945 & 0.8237 & 2330300 & 19945 \\
51. & coif4 & 0.9238 & 1789600 & 19945 & 0.8240 & 2328200 & 19945 \\
52. & coif5 & 0.9238 & 1791300 & 19945 & 0.8241 & 2327300 & 19945 \\
53. & dmey & 0.9237 & 1803000 & 19945 & 0.8246 & 2328400 & 19945 \\
\hline & & & & & & &
\end{tabular}

The sparsity analysis is performed with different sparsity measures such as Gini Index, L1-norm and L2-norm. The Table 3 shows the signal sparsity analysis of Discrete Wavelet Transform (DWT) family for 2-D Image (Example. Brain MRI).

The Table 3 shows that the bior3.7 (1-level decomposition) exhibits highest Gini index (GI=0.8347). It is also observed from the Table 3 that the bior3.1 exhibits the lowest L1-norm (2292600) and the bior5.5 shows the minimum L2- norm (19570).

For the DWT with 3-level decomposition, the bior3.1 wavelet attains the highest Gini index (GI=0.9355). Further, the bior5.5 achieves the lowest L1-norm (1733900) along with the lowest L2-norm (19102).

The Table 4 shows the 2-D image sparsity analysis for the DCT, FFT, LPC and PCA using Gini Index, L1norm andL2-norm.

Table 4. A comparative analysis of 2-D image sparsity for DCT, FFT, LPC and PCA using Gini index, L1-norm and L2-norm (Example Brain MRI)

\begin{tabular}{|c|c|c|c|c|}
\hline $\begin{array}{l}\text { Sr. } \\
\text { no. }\end{array}$ & $\begin{array}{l}\text { Sparsifying } \\
\text { Transform }\end{array}$ & Gini index (GI) & L1-norm & L2-norm \\
\hline 1. & DCT & 0.9513 & 10439 & 18809 \\
\hline 2. & DWT(1-Level) & 0.8347 (bior3.7) & 2292600 (bior3.1) & 19570 (bior5.5) \\
\hline 3. & PCA & 0.9533 & 7079.6 & 7775.0 \\
\hline
\end{tabular}

The Table 4 shows that the PCA exhibits the highest Gini index (GI=0.9533). It is also observed that PCA shows the lowest L1-norm (7079.6) and L2- norm (7775). This indicates that the PCA will provide more sparsity for 2-D image compared to other sparsifying transforms.

\section{Conclusion}

In this research paper, we have presented an investigation on sparsity analysis of 1-D speech signal and 2-D image signal using different sparsity measurement metrics such as Gini Index, L1-norm and L2-norm. Furthermore, the Discrete Wavelet Transform (DWT) family, FFT, DCT, LPC and PCA are used as the sparsifying transforms. 
Following major conclusions can be drawn based on the investigation:

- The result shows that the dmey wavelet (1-level decomposition) and the bior3.7 wavelet (3-level decomposition) show the greatest value of Gini index. The higher value of Gini index signifies the higher sparsity level of speech signal. Additionally, the bior5.5 wavelet (1-level and 3-level decomposition) and the bior3.1 (3-level decomposition) demonstrate the lowest value of the L1-norm and L2-norm. The lowest value of the L1-norm and L2-norm exhibits the higher sparsity of speech signal.

- Additionally, the DCT exhibits largest Gini index compared to FFT, LPC and PCA for speech signal. Also, the lowest value of the L1-norm and L2-norm are shown by the DCT.

- For 2-D image signal, the result shows that the bior3.7 (1-level decomposition) and the bior3.1 (3-level decomposition) exhibit the highest Gini index. Additionally, the bior5.5 wavelet (1-level and 3-level decomposition) and the bior3.1 (1-level decomposition) demonstrate the smallest value of the L1-norm and L2-norm.

- Moreover, the PCA exhibits the highest Gini index along with the lowest L1-norm and L2-norm which signifies the higher sparsity of 2-D image.

- Thus, the best sparsity basis can be selected based on sparsity measures such as Gini index, L1-norm and L2-norm.

- Furthermore, the Gini index can be considered as more consistent and general sparsity measure because of its advantages such as normalization within a range of 0 to 1 , scale-invariant and independent of the total signal energy, compared to traditional norm metrics.

Signal sparsity is one of the basic and important features in compressed sensing (CS) and dictionary learning based applications. The sparse signal or compressible signal is the basic requirement for successful implementation of compressed sensing (CS) technique. Thus, sparsity may play an important role in different signal processing applications.

\section{References}

[1] Mishali, M., Eldar Y.: Reduce and Boost: Recovering Arbitrary Sets of Jointly Sparse Vectors. IEEE Trans. Signal Processing, vol. 56, no. 10, pp. 4692-4702 (Oct. 2008)

[2] Huo Chengfu, Zhang Rong, Yin Dong: Compression Technique for Compressed sensing Hyperspectral Images. Taylor \& Francis, International Journal of Remote Sensing, Vol. 33, No. 5, 1586-1604 (2012)

[3] Qu Xiaobo, Zhang Weiru, Guo Di, Cai Congbo, Cai Shuhui, Chen Zhong: Iterative Thresholding Compressed sensing MRI Based on Contourlet Transform. Taylor \& Francis, Inverse Problems in Science and Engineering, Vol. 18, No. 6, 737-758 (2010)

[4] Mairal J., Elad M., Sapiro G.: Sparse Representation for Color Image Restoration. IEEE Trans. Image Processing, vol. 17, no. 1, pp. 53-69 (Jan. 2008)

[5] Aharon M., Elad M.: Sparse and Redundant Modeling of Image Content using an Image-SignatureDictionary. SIAM J. Imag. Sci., vol. 1, no. 3, pp. 228-247 (2008)

[6] Aharon M., Elad M., Bruckstein A.: K-SVD: An algorithm for Designing Overcomplete Dictionaries for Sparse Representation. IEEE Trans. Signal Processing, vol. 54, no. 11, pp. 4311-4322 (Nov. 2006)

[7] Leung K., Stralen M., Nemes A., Voormolen M., Burken G., Geleijnse M., Cate F., Reiber J., Jong N., Steen A., Bosch J.: Sparse Registration for Three-Dimensional Stress Echocardio-Graphy. IEEE Trans. Med. Imaging, vol. 27, no. 11, pp. 1568-1579 (Nov. 2008)

[8] Blu T., Dragotti P., Vetterli M., Marziliano P., Coulot L.: Sparse Sampling of Signal Innovations. IEEE Signal Processing Mag., vol. 25, no. 2, pp. 31-40 (Mar. 2008)

[9] Goyal V., Fletcher A., Rangan S.: Compressive Sampling and Lossy Compression. IEEE Signal Processing Mag., vol. 25, no. 2, pp. 48-56 (Mar. 2008)

[10] O'Grady P., Pearlmutter B., Rickard S.: Survey of Sparse and Non-Sparse Methods in Source Separation. Int. J. Imag. Syst. Technol., vol. 15, Special Issue on Blind Source Separation and Deconvolution in Imaging and Image Processing, no. 1, pp. 18-33 (Jul. 2005)

[11]He Z., Xie S., Ding S., Cichocki A.: Convolutive Blind Source Separation in The Frequency Domain Based on Sparse Representation. IEEE Trans. Audio, Speech, Language Processing, vol. 15, no. 5, pp. 1551-1563 (Jul. 2007)

[12] Hurley N., Rickard S.: Comparing Measures of Sparsity. IEEE Transactions on Information Theory, Vol.55, No.10, pp. 4723-4741 (October 2009) 
[13] Kassim Liban, Khalifa O., Gunawan T.S.: Evaluation of Sparsifying Algorithms for Speech Signals. In: International conference on computer and communication engineering (ICCCE 2012), Kuala Lumpur, Malayia, pp.308-313 (July 2012)

[14] Donoho D. L.: Compressed sensing. IEEE Trans. Inf. Theory, 52, (4), pp. 1289-1306 (2006)

[15] Baraniuk R. G.: Compressive sensing [Lecture Notes], IEEE Signal Processing Magazine, 24, (4), pp. 118121 (2007)

[16] Dalton H.: The Measurement of the Inequity of Incomes. Econom. J., vol. 30, pp. 348-361 (1920)

[17] Rickard S., Fallon M.: The Gini Index of Speech. In: Proc. Conf. Inf. Sci. Syst., Princeton, NJ (Mar. 2004)

[18]Lorenz M. O.: Methods of Measuring Concentrations of Wealth. J. Amer. Stat. Assoc. (1905)

[19] Gini C.: Measurement of Inequality of Incomes. Econom. J., vol. 31, pp. 124-126 (1921)

[20] Aaberge R.: Erratum to Axiomatic Characterization of the Gini Coefficient and Lorenz Curve Orderings. J. Econom. Theory, vol. 140, no. 1 (2008)

[21] Milanovic B.: A Simple Way to Calculate the Gini Coefficient, and Some Implications. Econom. Letter, vol. 56, no. 1, pp. 45-49 (1997)

[22] Moghadam Abdolreza, Hayder Radha: Hybrid Compressed sensing of Images. In: International workshop on multimedia signal processing (MMSP), Vol.99, pp.99-104 (2010)

[23]Zhou Jianqin, Chen Ping: Generalized Discrete Cosine Transform. Pacific-Asia Conference on Circuits, Communications and Systems, pp.449-452 (May 2009)

[24] Mallat Stephane: A Wavelet Tour of Signal Processing-The Sparse Way. Third edition, Academic Press (2009)

[25] Meyer Y.: Wavelets: Algorithms and Applications. Society for Industrial and Applied Mathematics, Philadelphia, pp. 13-31, 101-105 (1993)

[26] Daubechies Ingrid: Ten lectures on wavelets. CBMS-NSF conference series in applied mathematics SIAM Ed. USA (1992). http://dx.doi.org/10.1137/1.9781611970104

[27] Vaidyanathan P. P.: The Theory of Linear Prediction. Synthesis Lectures on Signal Processing, Morgan and Claypool Publishers.

[28] Giacobello Daniele, Christensen M. G., Murthi Manohar N., Jensen S. H., Moonen Marc: Retrieving Sparse Patterns Using a Compressed sensing Framework: Applications to Speech Coding Based on Sparse Linear Prediction. IEEE Signal Processing Letters, Vol. 17, No. 1, pp. 103- 106 (January 2010)

[29] Giacobello Daniele, Christensen Mads G., Murthi Manohar N., Jensen S. H., Moonen Marc: Sparse Linear Prediction and Its Applications to Speech Processing. IEEE Transactions on Audio, Speech, and Language Processing, Vol. 20, No. 5, pp. 1644-1657 (July 2012)

[30] Jackson, J. E.: A User's Guide to Principal Components, John Wiley and Sons, p. 592 (1991)

[31] Jolliffe, I. T.: Principal Component Analysis, 2nd edition, Springer (2002)

[32] Khan Zohaib, Shafait Faisal, Mian Ajmal: Joint Group Sparse PCA for Compressed Hyperspectral Imaging. IEEE Transactions on Image Processing, Vol. 24, No. 12, pp. 4934- 4942 (December 2015)

[33] University of Edinburgh (2002) Center for speech technology research, CSTR US KED TIMIT. URL: http://festvox.org/dbs/dbs_kdt.html. 